\title{
ON REFERENCE STAR RECOGNITION AND IDENTIFICATION
}

\author{
Ansis ZARIN̦Š ${ }^{1}$, Inese JANPAULE ${ }^{2}$, Jānis KAMINSKIS ${ }^{3}$ \\ Institute of Geodesy and Geoinformatics, University of Latvia, \\ Raina bulvāris 19, LV-1586, Riga, Latvia \\ E-mails: ${ }^{1}$ ansiszx@inbox.lv (corresponding author),2inesej@inbox.lv,3janis.kaminskis@rtu.lv
}

Received 6 November 2014; acceped 28 November 2014

\begin{abstract}
The paper deals with a research in the area of automation of positional star observations. In order to fully employ recent progress in imaging technologies, star image recognition and reference star identification process should gain comparable level of automation. A software package for this purpose has been developed in the Institute of Geodesy of the University of Latvia. It is capable of near-real-time image processing, star identification and astrometric position determination.
\end{abstract}

Keywords: geodetic astronomy, astrometry, star identification, image processing.

\section{Introduction}

Star positions long have been and still remain classical embodiment of a natural spatial reference system. Being easily accessible, well defined, accurately measurable, thoroughly researched, these positions allow to determine accurate spatial orientation in a number of different tasks and situations, ranging from spaceflight to geodynamic measurements. Recent progress in imaging technologies, though, has radically changed properties of star position measurement process. Capturing image on CCD matrices is incomparably simpler and faster than photographic methods, provides better sensitivity, and allows immediate astrometric processing of results. Also, astrometric coordinates of stars recently have got substantial increase both in accuracy and in numbers (Høg et al. 2000; Zacharias et al. 2005, 2013). To fully employ benefits from CCD technologies and extensive amount of accurate reference star positions, real-time processing must be supported, including automatic recognition of star images and their identification with reference catalog stars.

This paper reflects results of research, conducted in the interests of projects, involving astrometric image processing for device orientation determination in real time. Although the procedure can be used in wide variety of circumstances, presently our main areas of application are determination of camera orientation and determination of astrometric coordinates of an object.

Star recognition and identification on CCD images have somewhat different circumstances than for photographic images. The stage of recognition was generally done manually with photographic images; now differentiation of star and object images from CCD artifacts and background noise, determination of their boundaries, calculation of CCD coordinates of the center of image must be done automatically. In the stage of identification the most different aspect besides necessity to do everything automatically in our case is relatively small field of view, supported by CCD, containing, though, fainter and more numerous reference stars than it was common previously. Besides, it appears beneficial to have less strict requirements on preliminary frame position and orientation, our intention was to ensure identification in situations, when preliminary frame position accuracy is comparable to frame size and there is no information on frame orientation. Consequently, amount of candidate stars can become quite big, accordingly increasing identification difficulties.

Different aspects of digital astrometric image processing are described in numerous papers (e.g., Bertin, Arnouts 1996; Kouprianov 2007). However, 
for our task, as it turned out, a more robust and in some aspects simplified approach had to be realized to support automatic and near-real-time processing.

\section{Image properties}

Processing of astrometric images heavily depend on their specific properties. These images contain bright, but very small objects on generally dark background. However, due to high CCD sensitivity, background is well within captured intensity limits, especially for long exposures.

In terms of image content sources, astrometric images consist of:

- CCD electronic noise. It is growing with exposure time, for relatively short exposures normally does not exceed a few intensity bits, except some "hot pixels", for which readings can have any value,

- sky background - has generally Gaussian distribution, part of it can be beyond imager sensitivity; there is no use of astrometric images with saturated background, so we can assume that the bright end of background distribution is well represented. Dispersion of background distribution can be small (a few intensity bits), resulting in very narrow graph,

- star images - bright, but small. Images of the brightest stars often saturate sensor, making maximum intensity image kernel. Images are surrounded by secondary diffraction maximum rings, resulting in spots (in ideal circumstances - circular, in reality - always distorted to some extent by a number of factors - convection, fundament vibrations, defects or misadjustment of optics, tracking errors; are elongated if telescope is not tracking stars) with radially dropping intensity; size (summary pixel intensity) is proportional to star magnitude,

- occasional artifacts, caused by other objects within field of view, reflections from construction elements, light scattering in media, electronic artifacts of image read-out process.

Resulting pixel intensity distribution has 3 main components:

- hardware noise - normally has low intensity, except in special conditions like long exposure, high temperature,

- background illumination - generally uniform within frame, but can contain large-scale patterns, caused by nearby light sources; distribu- tion locally close to Gaussian, the weakest part of it can be missing,

- star images - superposition of many nearGaussian distributions with various peak intensities (up to saturated), but little pixel count. In order to be automatically and with certainty distinguishable from background, only those with peak intensity noticeably above background peak are of interest.

As the part of image information, representing background, is of little interest for positioning purposes, it is often removed during image processing (after background flattening), linearly transforming pixel intensity so, that full intensity range represents original intensities from peak of background intensity to saturation (or some maximum intensity, if saturation is not present or saturated images are dismissed from processing). That has to be done for visualization of images with bit count above 8 , for most visualization media do not support bigger intensity range. Resulting image has generally black background and improved contrast.

For narrow field of view ( $<\sim 0.5 \mathrm{dg})$ background intensity in most cases is nearly uniform throughout the frame, not-negligible background pattern appears only in vicinity of light sources (Moon, illuminated clouds, lamps). We found that a simple and efficient substitute to calculating background map for whole frame is a second iteration in background intensity estimation, using only vicinity of each object for background intensity estimation instead of whole frame.

\section{Image recognition}

For positioning purposes, we are dealing mostly with short (sub-second) exposures, so that star images are only slightly elongated. That allows greatly simplify image recognition and image center calculation algorithms.

Implemented star image recognition algorithm is based on detecting compact groups of pixels, brighter than background. The threshold pixel intensity, determining adherence to an image, may be defined as the intensity at which near-Gaussian background distribution gives way to superimposed distributions representing brighter, but much smaller in size, star images, forming intensity distribution tail (Fig. 1). This definition is rather vague, so a practical, albeit not strictly based criteria is intensity, above which background distribution contains small enough number of pixels. 
Smallness can be measured in terms of relative number of pixels on CCD - one background pixel above threshold level in about a thousand ( 2.3 dispersions from mean of distribution) often is an acceptable concentration of background, little interfering with star images.

Background distribution parameters must be determined to estimate such threshold level value. As there is no guarantee, that whole background intensity range will be present in data and presence of other image components deforms the distribution, which in itself is not strictly Gaussian, distribution parameters can be estimated only approximately. One of possible algorithms includes determination of distribution maximum position (taking for granted, that maximum is represented in image), then determination of dispersion using data portions, symmetric to maximum.

As different parts of frame can experience slightly different background intensity, it may be reasonable to adjust threshold level to vicinity of a particular star in the final stages of star recognition.

Local intensity maximums - pixels, surrounded by less or equal intensity neighbors, are used as "seed" points for star images. They are found traversing nondecreasing sequences of pixels. Each traversed pixel is marked in order to prevent subsequent traverses of stepping on already visited area. This way, several maximums can be found for areas with uniform intensity, they must be joined during further analysis of image.
As the next step, image area is formed around each maximum point, assigning to it adjoining pixels above intensity threshold level (Fig. 2). Overlaying or touching image areas can be joined together, however, that includes risk of merging images of several objects. An opposite effect - potential fragmentation - can occur if image area is limited to equal or decreasing
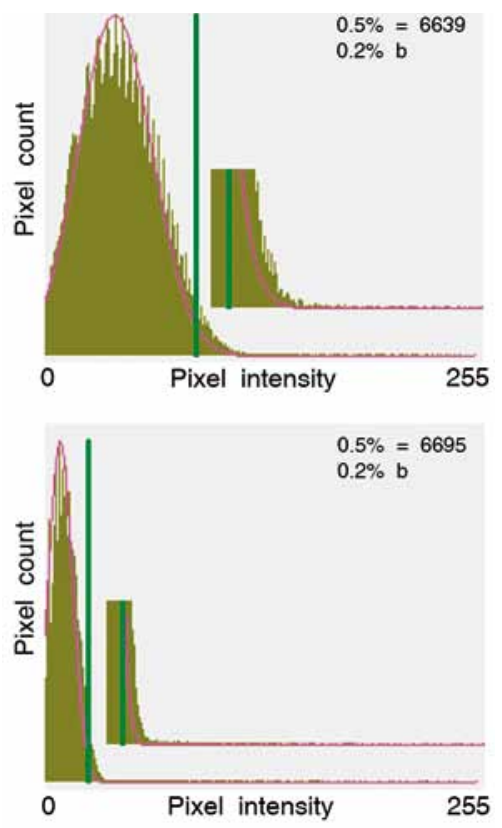

Fig. 1. Frame pixel intensity distribution examples with different background intensity

Notes: Background threshold level selected to leave $0.2 \%$ of background distribution pixels. In both cases that means $\sim 0.5 \%$ of all pixels, but that percentage can be different depending on star distribution density and presence of artifacts.

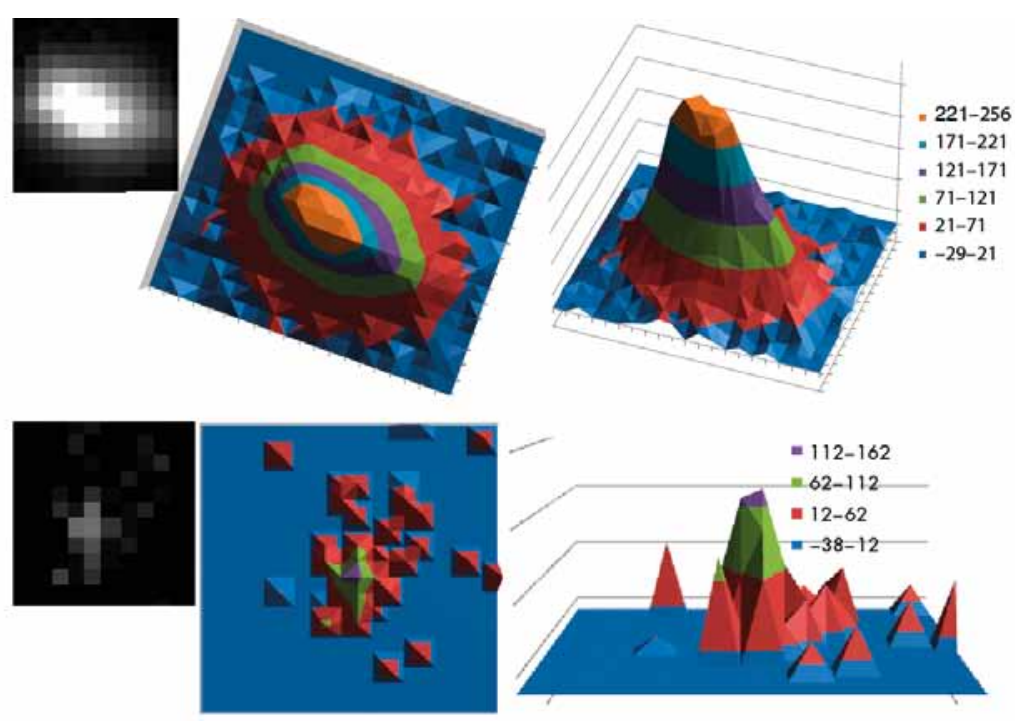

Fig. 2. Image analysis for a bright and a weak star

Notes: Pixels above and including the green band are assigned to images. Slight saturation of sensor present in the bright image. Image elongation due to 0.4 second exposure. 
intensity neighbors, rejecting secondary maximums. To remain flexible, control of image forming tactics is available to operator and it can be changed according to current objective.

Such simplified approach can be not adequate in case of long exposures or fast-moving objects, when long image trails are present. In such circumstances more detailed image analysis (PSF fitting or similar) should be performed.

Mass (intensity) center of image is used as the initial point of coordinate calculations. For centrally symmetric images it is quite well defined and coordinates almost independent of object's brightness. However, for distorted or elongated images, such definition of image center becomes vague, brightness dependency can be present. In particular, poorly adjusted optics can introduce brightness dependency of position up to several pixel size. That again calls for more detailed image analysis.

\section{Identification}

Defining target properties for identification procedure, we agreed on following:

- frame's preliminary position must be known at least with frame size accuracy (in our case that is about a half of degree of arc ), so we can guarantee that frame area is included in reference star selection buffer zone with radius equal to the frame size,

- frame scale is known with at least $\sim 10 \%$ accuracy. Refocusing can change focus distance in the process, so some uncertainty may be present, especially after changes in hardware components,

- CCD attachment can introduce quite big uncertainties in frame rotation position, so, to be on the safe side, it is preferable not to make any assumptions on frame rotation - any position can be expected,

- star image magnitudes can be (before identification) estimated with a few magnitude unit accuracy, so catalog star pairing with star images can be somewhat (but not very strictly) limited,

- success of identification is recognized by presence of some minimal number (typically $\sim>5$ ) of identified stars, producing small enough frame model approximation rms (value of this limit should be connected to average accuracy of system),
- for identification purposes it is enough to regard only limited number $(<10 \ldots 30)$ of brightest star images, if there are more, their identities can be added later, when a reasonable frame model is available,

- to have real-time data processing, calculation time should not exceed characteristic time of other involved operations. In our case, the longest time is needed for image read-out and transfer to computer, typically a few seconds. So, in most cases identification time should not much exceed one second.

In principle, identification of 2 stars is enough to determine frame model (position, orientation and scale). That makes possible a trivial procedure - loop through all pairs of two star images and two catalog stars (starting from brightest), checking if frame model, based on them, allows picking up of more catalog stars which are close enough to other star images. With proper identification criteria it works in majority of cases, but can consume unacceptably much time - typically minutes, can take hours if numbers of involved images $(\sim>10)$ and candidate stars $(\sim>100)$ are big, and can lead to false identification, if minimum identified stars number limit is low. While usable as a last resort when nothing else works and there is enough time, such method is not fit for real-time mode.

Another trivial procedure is possible when position and orientation of frame is well-known and allows immediate picking up of proper catalog stars. It is efficient in processing a series of frames obtained without moving telescope or while tracking a star, or if telescope pointing and orientation is otherwise precisely known, but is of no use in a general situation.

As proved in earlier researches, more efficient method is based on comparison of geometrical properties of figures, formed by three reference stars and three star images: vertex angle and proportion of edge lengths. Properties of such identification algorithm are discussed in another paper of this journal (Balodis et al. 2014).

\section{Test results}

An implementation of algorithms described above was tested on star observation frames from digital zenith camera (Abele et al. 2012 assessment sessions. Images were acquired with a $200 \mathrm{~mm}$ catadioptric telescope (focus distance $1400 \mathrm{~mm}$ ) using 1.3 Mpix CCD with $6.45 \mu$ pixel size (image scale close to 1 " per pixel, 
frame size $0.26 \times 0.36 \mathrm{dg}$ ). At 0.2 second exposure frame typically contained images of $10 \ldots 60$ stars of magnitude up to $13^{\mathrm{m}}$. A subset of NOMAD catalog (Zacharias et al. 2005) was used for reference star data; apparent star coordinates were calculated using NOVAS astrometric package (Kaplan et al. 2005); alternatively, STARLINK package (Disney, Wallace 1982) was tested and gave practically identical results. Reference star candidates were selected within circular area, centered on predicted frame position, with radius of frame diagonal. Depending on proximity of Milky Way, 100-2000 candidate stars were present in selection area.

After establishing optimal set of parameters, star recognition and identification was successful for practically all test frames - only a few out of about 1000 failed. The main reason of failures was insufficient number of star images within frame - less than 6-7, so that results did not qualify because of too little number of found identities. Identification time generally was between 0.05 and 0.2 seconds, with some cases of 2-3 seconds. Together with other associated calculations (data input/output, image analysis, star catalog access), average processing time was close to 2 seconds per frame. We find such results quite satisfactory. Astrometric processing module is now being integrated in several real-time data acquisition and device control software packages.

\section{Acknowledgement}

This research was funded by ESF, project No 2013/0066/1AP/1.1.1.2.0/13/APIA/VIAA/059.

\section{References}

Abele, M.; Balodis, J.; Janpaule, I.; Lasmane, I.; Rubans, A.; Zariņš, A. 2012. Digital zenith camera for vertical deflection determination, Geodesy and Cartography 38 (4): 123-129. http://dx.doi.org/10.3846/20296991.2012.755324

Balodis, J.; Zariņš, A.; Haritonova, D.; Janpaule, I. 2014. Parameters for automated star identification, Geodesy and Cartography 40(4): [this issue] http://dx.doi.org/10.3846/20296991.2014.987457

Bertin, E.; Arnouts, S. 1996. SExtractor: software for source extraction, Astronomy and Astrophysics Supplement Series 117: 393-404. http://dx.doi.org/10.1051/aas:1996164

Disney, M.; Wallace, P. 1982. Starlink, Quarterly Journal of the Royal Astronomical Society 23: 485-504.

Høg, E.; Fabricius, C.; Makarov, V. V.; Urban, S.; Corbin, T.; Wycoff, G.; Bastian, U.; Schwekendiek, P.; Wicenec, A. 2000. The Tycho-2 Catalogue of the 2.5 million brightest stars, Astronomy and Astrophysics 355: 19-22.
Kaplan, G. 2005. The IAU resolutions on astronomical reference systems, time scales, and Earth rotation models explanation and implementation, United States Naval Observatory circular No. 179. Washington, DC: U.S. Naval Observatory.

Kaplan, G.; Hughes, J.; Seidelmann, P.; Smith, C.; Yallop, B. 1989. Mean and apparent place computations in the new IAU system. III. Apparent, topocentric and astrometric places of planets and stars, The Astronomical Journal 97(4): 1197-1210. http://dx.doi.org/10.1086/115063

Kouprianov, V. 2007. Distinguishing features of CCD astrometry of faint GEO objects, Advances in Space Research 41(7): 1029-1038. http://dx.doi.org/10.1016/j.asr.2007.04.033

Zacharias, N.; Finch, C. T.; Girard, T. M.; Henden, A.; Bartlett, J. L.; Monet, D. G.; Zacharias, M. I. 2013. The fourth U.S. Naval Observatory CCD Astrograph Catalog (UCAC4), The Astronomical Journal 145, 44 [online], [cited 06 November 2014]. Available from internet: http://cdsarc.u-strasbg. fr/viz-bin/Cat?I/322

Zacharias, N.; Monet, D.; Levine, S.; Urban, S.; Gaume, R.; Wycoff, G. 2005. The Naval Observatory Merged Astrometric Dataset (NOMAD), in AAS 205 ${ }^{\text {th }}$ meeting, \#48.15, 11 January 2005, SanDiego, CA [online], [cited 06 November 2014]. Available from Internet: http://www.nofs.navy.mil/ nomad/

Ansis ZARIN̦Š. Dr, Leading researcher at the Institute of Geodesy and Geoinformation, University of Latvia, Raina bulv. 19, LV-1586 Riga, Latvia (Ph +371 67034435), e-mail: ansiszx@inbox.lv. (Dr phys. 1988).

Research interests: control and data processing systems for satellite observation and astrometric instruments.

Inese JANPAULE. Dr, leading researcher at the Institute of Geodesy and Geoinformation, University of Latvia, Raina bulv. 19, LV-1586 Riga, Latvia. Ph +371 67034435, e-mail: inesej@inbox. lv. Graduate of Riga Technical University (Dr sc. ing. 2014).

Research interests: GNSS, land surveying, Bernese GNSS software applications, geoid modelling.

Jānis KAMINSKIS. Dr, leading researcher at the Institute of Geodesy and Geoinformation, University of Latvia, Raina bulv. 19, LV-1586 Riga, Latvia. Ph +371 67034435, e-mail: Janis.Kaminskis@rtu.lv. Graduate of Riga Technical University (Dr sc. ing. 2010).

Research interests: geoid modelling, satellite geodesy, vertical deflection measurements. 